\title{
USE OF MULTIMOD ALITY IMAGING AND NEUROPSYCHOLOGICAL MEASURES FOR THE ASSESSMENT AND TREATMENT OF AUDITORY VERBAL HALLUCINATIONS: A BRAIN TO BEHAVIOR APPROACH
}

\author{
Elizabeth M. Zimmerman ${ }^{2}$, Megan A. Golla ${ }^{1,2}$, Renata A. Paciora ${ }^{1}$, Philip S. Epstein, ${ }^{1}$ \\ \& Lukasz M. Konopka ${ }^{1,2,3 *}$ \\ Advanced Clinical Neuroscience ", The Chicago School of Professional Psychology ${ }^{2}$, and Loyola University \\ Department of Psychiatry ${ }^{3}$, Chicago, Illinois, USA
}

\begin{abstract}
Patients suffering from auditory verbal hallucinations (AVHs) may utilize unique coping strategies in order to reduce distraction caused by the perception of internal speech. Behavioral strategies may include externalizing speech through self-talk. This paper examines the benefits of a multimodality brain to behavior approach applied to the outpatient psychiatric assessment of a 55-year-old Caucasian female presenting with auditory verbal hallucinations and challenges in attention, concentration, and emotional regulation, who employed self-talk as a coping strategy to focus her attention.
\end{abstract}

Key word s: EEG; hallucinations; LORETA; SPECT; neuropsychology

\section{INTRODUCTION}

Convergent quantitative EEG (LORETA), quantitative SPECT, and neuropsychological data are presented in order to highlight the need for objective, individualized assessment and treatment of patients with complex clinical presentations. The comprehensive assessment revealed several aberrant findings, which help to explain the patient's presenting symptoms. Treatment considerations are also presented.

\section{CASE REPORT}

The patient is a 55-year-old, right-handed Caucasian female, who presented for outpatient evaluation with the primary complaints of auditory verbal hallucinations (AVHs) and difficulty with attention, concentration, and emotional regulation, all of which had been chronic issues for the past 15 years. At the time of neuropsychological assessment, she reported she could hear her "other personalities talking to each other inside [her] head."

The patient reported problems with reduced productivity at several jobs in recent years due to memory gaps, difficulty telling time, and a lack of attention and concentration. She was unemployed at the time of evaluation. Her primary healthcare physician had previously diagnosed the patient with Attention Deficit/ Hyperactivity Disorder according to the DSM-

"Correspondence to: Lukasz M. Konopka, e-mail: lkonopka@thechicagoschool.edu

Received July 20, 2011; accepted September 20, 2011; Act Nerv Super (Praha) 53(3-4),150-8. 
IV-TR (American Psychiatric Association, 2000). At the present evaluation, her working diagnosis was Dissociative Identity Disorder assigned by her previous therapist. She was referred for a neuropsychological evaluation accompanied by neuroimaging. The patient gave written, informed consent for involvement in both therapy and research.

The patient denied any history of traumatic brain injury or seizures. She has a history of sexual and physical abuse that began at the age of 4 and continued throughout her adult years. She reported a long history of emotional dysregulation. The patient explained that, prior to 2008, she engaged in self-cutting up to three times per week and was preoccupied with rejection and abandonment by others. She denied current self-harm.

Between the ages of 15 and 26, the patient used a variety of illicit substances including alcohol, amphetamines, barbiturates, cannabis, cocaine, hallucinogens, and opiates. The patient had completed more than 15 years of formal education at the time of evaluation, throughout which she attained variable grades ranging from A's to F's reportedly due to problems with attendance and inconsistent levels of motivation.

On the day of evaluation, the patient reported her fasting blood sugar was $90 \mathrm{mg} / \mathrm{dl}$. She listed the use of the following medications: Cinnamon (500mg q.d.), Vyvanse (60 mg q.d.), Omega 3 supplements (900mg BID), Vitamin D Drops (q.d.), and Centrum Silver Vitamin (q.d.). The patient denied any illicit drug use at the time of evaluation.

\section{BRAIN IMAGING METHODS}

Localization of neurological activity was measured with neuroimaging techniques including quantitative Electroencephalography (qEEG) with Low Resolution Brain Electromagnetic Tomography (LORETA) and quantitative Single Photon Emission Computerized Tomography (qSPECT). The patient completed a qEEG, followed one month later by qSPECT and finally a neurobehavioral evaluation three months after her qEEG.

EEG was recorded in the alert resting state with eyes closed. Digital EEG was acquired from 19 cephalic electrodes applied based on the International 10/20 System. Silver silverchloride electrodes were used. Four non-cephalic electrodes were applied to monitor EKG as well as eye movements. The ear lobes had active leads. Linked ears reference was computed from the active leads and used in subsequent analyses. The electrode impedance was $<5$ $\mathrm{kOhms}$ and was checked throughout the recording. The acquisition-sampling rate was at 500 $\mathrm{Hz}$ and the filter settings were at 0.015 and 70 .

During the EEG recording, the patient was seated in a comfortable chair in a quiet room with an ambient temperature of about $23^{\circ} \mathrm{C}$. The data analysis of qEEG was performed using NeuroGuide V2.1.1 EEG software.

qEEG objectively measures electrical activity of the brain and statistically compares this activity to a normative sample based on age, gender, and handedness (Thatcher et al., 2003). The point of maximal activity is then localized with low resolution brain electromagnetic tomography (LORETA) to identify more precise areas of activation within 3-D MRI space. Findings were considered significant at the level of \pm 2.0 standard deviations. NeuroGuide's normative database set the comparison from which we evaluated normality.

The brain SPECT study was performed during a baseline period whereby the patient was injected with a $26.1 \mathrm{mCi}$ dose of technectium-99m-HMPAO (Amersham International) without complication. Again the patient was seated in comfortable chair in a quiet room with an ambient temperature of about $23^{\circ} \mathrm{C}$ during the SPECT study. SPECT imaging data acquisition occurred approximately 90 -minutes after the injection of the HMPAO ligand (see Goforth et al., 2004). SPECT data acquisition was performed using a rotating gamma camera system equipped with ultra high-resolution fan beam collimators.

A visual inspection of unprocessed SPECT images was done by board certified nuclear medicine physician. Using the HERMES processing system (Nuclear Diagnostics, Hagerstad, Sweden), images were spatially and intensity normalized. Region of interest based template was used for statistical evaluation of the intensity measures using HERMES BRASS ${ }^{\mathrm{TM}}$ based 
methods (Nuclear Diagnostics, Stockholm, Sweden). Statistical maps were generated based on the comparison of the SPECT data between the patient and the normal controls developed by HERMES. The abnormality threshold was set to a $2.5 \mathrm{~mm}$ with a standard deviation of 3.5.

\section{NEUROBEHAVIORAL METHODS}

Neuropsychological functioning was assessed with the Repeatable Battery for the Assessment of Neuropsychological Status (RBANS), Integrated Visual and Auditory Continuous Performance Task (IVA+Plus), Cambridge Neuropsychological Test Automated Battery (CANTAB), and Trail Making Test A and B (TMT). All neurobehavioral measures were conducted on the same day. The patient was tested in a quiet room.

RBANS is a brief objective measure of broad neurop sychological functioning with norms based on age. This test contains 10 subtests that comprise five index scores and a total scale score. These index measures include immediate and delayed memory, visuospatialconstructional skills, language, and attention. RBANS provides an overall summary of broad neuropsychological functioning. Its effectiveness has been well documented as a cognitive screening tool for many psychological disorders (Dickerson et al., 2004).

IVA+Plus is a computerized continuous performance task that measures visual and auditory attention and response control (Kane \& Whiston, 2001). The test contains a normative group divided by age and gender. This test also indicates the presence of fine motor hyperactivity and highlights preferences for visual or auditory information encoding. Previous studies have suggested that patients with schizophrenia demonstrate better visual over auditory performance (Baerwald, Tyron, \& Sandford, 2001).

CANTAB is a comprehensive, non-verbal computerized cognitive test battery measuring six general indexes: Induction, Visual Memory, Executive Function, Attention, SemanticVerbal Memory, Decision Making and Response Control. The individual CANTAB tests are presented on a computer-controlled touch-screen monitor and are designed to assess specific brain regions, yielding a $\mathrm{z}$-score normed by age and gender. Induction tests were used prior to the administration of function-specific tests to control for abilities in comprehension and reaction time. The tests in the present battery assessed executive functioning and visual memory. One of these tests, the Stockings of Cambridge (SOC), measures executive functioning and is essentially a computerized version of the Tower of London Test. The patient is shown a display containing three different colored balls and is asked to make his/ her arrangement match the computer generated display in the fewest number of moves possible. For an overview of the other specific tests used in the current battery (Big Little Circle, Spatial Span, Intra/ Extradimensional Set Shift, and Paired Associates Learning), see Sweeney, Kmiec, and Kupfer (2000). For a detailed description of CANTAB tests, see Lawrence and Sahakian (1996) and Robbins et al. (1994).

The Trail-Making Test (TMT) is a brief measure of prefrontal lobe functioning involving such abilities as following brief instructions, sustaining attention, shifting cognitive sets, problem solving and planning (Reitan \& Wolfson, 1986; Lezak, 1995). Trails A requires mental processing speed, visual scanning, and sustained attention. Trails B requires the simultaneous alteration of attention between two response sets. Scoring for these tasks are recorded according to the time participants take to complete them. Higher times indicate greater impairment in neuropsychological functioning.

\section{RESULTS}

\subsection{Imaging findings}

Imaging results revealed notable convergent findings. A statistical comparison of the SPECT data between the patient and normal controls identified perfusion excess in the patient's left 
lateral temporal lobe (Figure 1A). The quantitative analysis of background EEG activity demonstrated statistically significant corresponding excess activity in alpha-2 frequency (11 $\mathrm{Hz}, \mathrm{z}=2.36)$ and a deficit of slow activity in theta frequency $(5 \mathrm{~Hz}, \mathrm{z}=-2.45)$.

\section{Figure 1A. Left Temporal Lobe Excess}

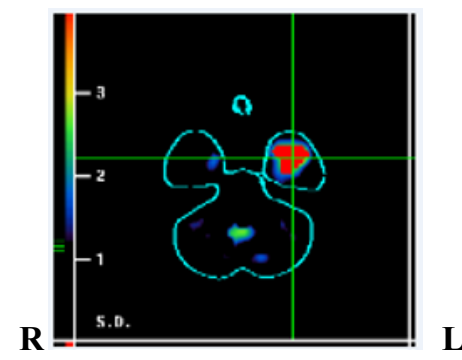

Statistical SPECT Excess Perfusion
$\mathbf{L}$

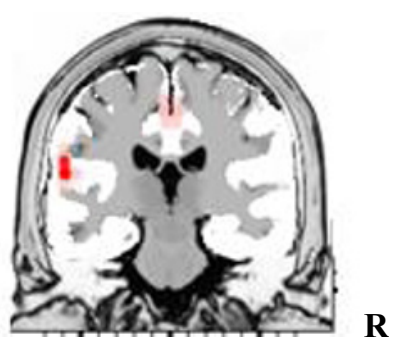

LORETA Excess in Alpha $2(11 \mathrm{hz})$

Figure 1B. Right Dorsal Lateral Frontal Deficit

$\mathbf{R}$
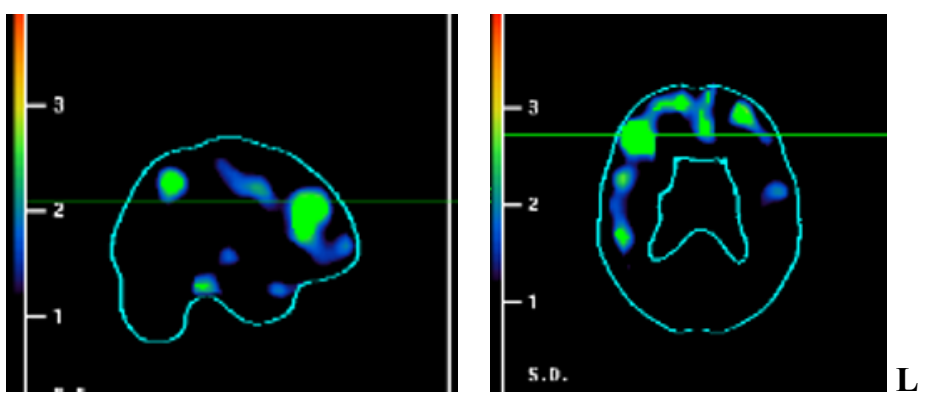

Statistical SPECT Perfusion Deficit

\section{Figure 1C. Right Temporal Lobe Deficits}

$\mathbf{R}$

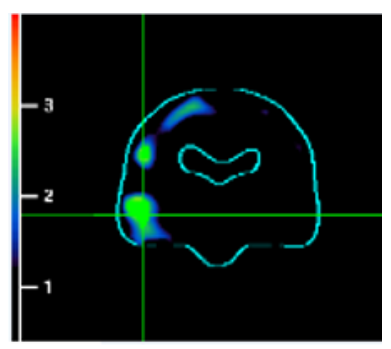

$\mathbf{L}$

Statistical SPECT Perfusion Deficit
$\mathbf{L}$

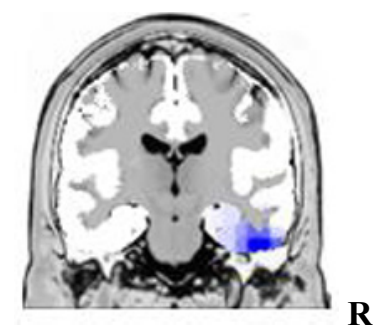

LORETA Deficit in Theta $(6 \mathrm{hz})$

Figure 1. Statistically significant findings in SPECT and LORETA.

Note: (A) Left: Perfusion excess in left lateral temporal lobe; Right: Excess alpha-2 frequency in superior temporal gyrus at $11 \mathrm{~Hz}, z=2.36, B A 38$. (B) Perfusion deficit in right posterior dorsal frontal cortex. (C) Left: Perfusion deficit in right lateral temporal lobe; Right: Deficit in theta frequency in right inferior temporal gyrus at $6 \mathrm{~Hz}, \mathrm{z}=-2.53, \mathrm{BA} 20$.

Through LORETA, we localized the source of aberrant activity of excess beta to the superior temporal gyrus (Brodmann area 38; Figure 1A) and deficit in theta to the left inferior 
temporal gyrus (Brodmann area 20). In SPECT findings alone, additional areas of statistical significance include hypoperfusion in the right posterior dorsal frontal cortex (Figure 1B). EEG did not have correlate findings to this area of deficit. SPECT also revealed a deficit in perfusion in the right lateral temporal lobe, overlapping with EEG deficit in theta $(6 \mathrm{~Hz}, \mathrm{z}=$ 2.53). This deficit was localized in LORETA to the right inferior temporal gyrus, Brodmann area 20 (Figure 1C).

\subsection{Neurobehavioral findings}

The patient's performance on the IVA+Plus revealed extreme deficits in auditory attention (See Table 1). The patient obtained a Sustained Auditory Attention Quotient of 34 points, which is a large discrepancy compared to her Sustained Visual Attention Quotient of 104 points. This discrepancy highlights the patient's preference for visual processing and encoding over auditory processing. These auditory deficits are likely due to the patient's reported auditory verbal hallucinations $(\mathrm{AVH})$.

Table 1. IVA+Plus data with performance descriptors.

\begin{tabular}{|l|l|}
\hline INDEX & SCORE \\
\hline Sustained Auditory Attention & $\mathbf{3 4}$ \\
& Extreme Deficit \\
\hline Sustained Visual Attention & $\mathbf{1 0 4}$ \\
Average
\end{tabular}

Note. IVA+Plus has a mean of 100 and a SD of 15.

Table 2. CANTAB data with performance descriptors. The results are ind icated in $\mathrm{z}$-scores.

\begin{tabular}{|c|c|c|c|c|c|}
\hline \multirow{2}{*}{$\begin{array}{l}\text { CANTAB } \quad \text { Test } \\
\text { Category }\end{array}$} & \multirow[t]{2}{*}{ CANTAB Test Performed } & \multirow{2}{*}{$\begin{array}{l}\text { Standard } \\
\text { Score }\end{array}$} & \multicolumn{3}{|c|}{ Performance Description } \\
\hline & & & $\begin{array}{l}\text { Below } \\
\text { Average }\end{array}$ & Average & $\begin{array}{l}\text { Above } \\
\text { Average }\end{array}$ \\
\hline \multirow[t]{3}{*}{$\begin{array}{l}\text { EXECUTIVE } \\
\text { FUNCTION, } \\
\text { WORKING } \\
\text { MEMORY, AND } \\
\text { PLANNING } \\
\text { TEST }\end{array}$} & \multirow[t]{3}{*}{$\begin{array}{l}\text { Stockings of Cambridge } \\
\text { (SOC) }\end{array}$} & $\begin{array}{l}\text { Mean Initial } \\
\text { Thinking } \\
\text { Time } \\
\text { moves) } \\
-2.11\end{array}$ & $\mathbf{X}$ & & \\
\hline & & $\begin{array}{l}\text { Mean } \\
\text { Subsequent } \\
\text { Thinking } \\
\text { Time } \\
\text { Moves } \quad(5 \\
1.19 \\
\end{array}$ & & & $\mathrm{X}$ \\
\hline & & $\begin{array}{l}\text { Problems } \\
\text { Solved in } \\
\text { Minimum } \\
\text { Moves } \\
1.09\end{array}$ & & & $\mathrm{X}$ \\
\hline
\end{tabular}


In terms of executive functioning, working memory, planning, and visual memory as measured by CANTAB, the patient was functioning in the average to above average range (Figure 6). On the Stocking of Cambridge subtest, the patient took extra time to audibly verbally plan each move and discuss her strategy before engaging in the task.

This process proved effective for the patient in regards to problem solving; however, selftalk negatively affected her processing speed and resulted in a lower score. (See Table 2). Additionally, the patient continued to audibly verbalize her answers to herself throughout the assessment process. All other neuropsychological test results were within normal limits.

\section{DISCUSSION}

The results of the patient's neuroimaging and neuropsychological tests verified underlying biological constructs that play a key role in her symptomatology. Convergent deficits in SPECT and EEG revealed focal abnormalities in the left temporal lobe. Such focal abnormalities found across modalities implicate the importance of left temporal lobe involvement in the patient's clinical presentation. These results are supported by previous imaging studies of AVHs. Additionally, aberrant activity in the patient's right dorsolateral prefrontal area as identified by SPECT implicate this region in the patient's difficulties with attention and concentration.

\subsection{Auditory Verbal Hallucinations}

Auditory hallucinations are correlated with increased activity in auditory-processing netw orks (Ford et al., 2009; van den Ven et al., 2005; Lennox et al., 2000; McGuire, Silberweig, \& Frith, 1996; Tiijhonen et al., 1992). Previous SPECT studies of AVHs focus on patients diagnosed within the schizophrenia disorder spectrum. However, Mauri et al. (2008) reported hyperperfusion in the left temporal cortex in patients with AVHs who, similarly to our patient, do not have additional symptoms of schizophrenia. In addition, Bentaleb et al. (2002) demonstrated findings of a woman with continuous AVHs that stopped when she listened to external speech. Bentaleb et al. (2002) reported elevated metabolic activity in the left primary auditory cortex in the absence this external speech. Although neuroimaging of our patient while talking is unavailable, she did demonstrate increased perfusion to auditory processing areas in the left hemisphere during rest and she used self-talk throughout neuropsychological testing as a method to improve concentration.

Previous EEG to studies of AVHs have indicated similar patterns of focal activity. Ishii and colleagues (2000) identified a strong association between activity of the superior temporal gyrus and hallucinations. Spencer et al. (2009) suggest that hallucinations are related to cortical hyperexcitability, and similarly to SPECT studies, point to language-related areas of the left hemisphere. Supporting previous studies, our patient demonstrated convergent focal abnormalities in EEG and SPECT localized to the left temporal gyrus, demonstrating a deficit in theta frequencies $(5 \mathrm{~Hz})$ and excess in alpha-2 $(11 \mathrm{~Hz})$ as well as significantly increased perfusion in the left lateral temporal lobe.

Results of the patient's neurobehavioral assessment support the results in the literature regarding auditory performance. Significant findings were identified with in IVA whereby the patient demonstrated extreme deficits in auditory attention, while neuropsychological findings of attention and concentration were within normal limits. This discrepancy highlights the patient's difficulty with encoding verbal information, likely due to her reported AVHs. The multimodal neuroimaging results confirm the patient's disrupted auditory networks. Results in qSPECT indicated perfusion abnormalities in the left lateral temporal lobe and overlapping EEG excess beta in the superior temporal gyrus. Thus, the neuroimaging abnormalities support the localization of the patient's clinical auditory 
abnormalities, highlighting the sensitivity of neuroimaging tools to specific clinical presentation.

\subsection{Attention and Concentration}

Many areas of our patient's aberrant brain activity that relate to auditory hallucinations are also involved in attentional processes. Amen and Carmichael (1997) identified decreased perfusion in the prefrontal cortex both at rest and during cognitive tasks, with an increase in left temporal lobe activity. Kim et al. (2002), using statistical parametric mapping, reported decreased blood flow in the right prefrontal and right middle temporal cortices in 40 children with ADHD. In a SPECT study of impulsivity-related personality disorders such as Borderline Personality Disorder and Antisocial Personality Disorder, Goethals et al. (2005) reported similar areas of decreased $\mathrm{rCBF}$ in the right prefrontal cortex as well as the right lateral temporal cortex, suggesting that these areas are relating to the impulsive component of certain impulsive-personality disorders. Impulsivity, a central characteristic of ADHD, may also overlap with the patient's behaviors of inattention and lack of concentration. Thus, while the neuropsychological assessment failed to fully verify reported symptoms, neuroimaging results validate the patient's reported difficulties with attentional difficulties that, prior to treatment, had caused significant occupational impairment.

\subsection{Limitations}

While our patient was assessed across modalities to aid in diagnosis and treatment, our study would be more powerful if SPECT and EEG were performed simultaneously and immediately following a neurobehavioral assessment. Instead, the patient had SPECT approximately one month after an EEG test and approximately two months before neurop sychological testing (Poprawksi et al., 2007). Because of the span of time between each assessment, SPECT, EEG, and neuropsychological data may be under-representative of convergent patterns of activation. Areas of activity may have changed based on temporary states. Also, the database for z-score estimation in qEEG and qSPECT are different, limiting the statistical correlation between the two measures.

Another weakness of this study is that the patient was taking several medications at the time of brain imaging, which could also alter both imaging and neuropsychological results. Additionally, it would be beneficial to have our patient perform a task, such as TMT, during SPECT in order to see if normalization of perfusion would occur in the auditory processing areas in the left hemisphere in the presence of external speech. Finally, while case studies can be useful examples, the generalizability of our results is limited.

\section{CONCLUSION}

In summary, with a multimodal approach to diagnosis and treatment, clinicians can identify the involvement of different brain networks allowing more specific designs to therapeutic intervention. Such an approach is likely to reduce costs and duration of symptoms and treatment. qEEG as a modality is widely available, transportable, inexpensive, and noninvasive, and it is based on a normative sample (Thatcher et al., 2003). This modality also has confirmed cross-cultural specificity and replicability (Hughes \& John, 1999; Congedo \& Lubar, 2003). SPECT, although more costly, can provide information about brain perfusion to correlate with electrical activity and further the accuracy of localization and severity of abnormality. By correlating these modalities with neuropsychological testing data, clinicians can assess direct behavioral outputs based on brain patterns and utilize clinical judgment to focus on areas most in need of intervention. 
Our patient presented with a complex psychiatric history and had been in treatment for several years prior to these neuropsychological and neuroimaging studies. Without objective assessment measures, previous mental health professionals had attempted to treat her using talk therapy. This approach is inherently problematic for our patient because of her impaired ability to process auditory information. Objective neuroimaging and neuropsychological measures, however, revealed that the patient's attentional networks and language-processing networks showed aberrant activation patterns, explaining why she did not benefit from listening to a therapist. After these studies, the patient's treatment providers altered approaches to therapy and medication, incorporating neuroimaging and neuropsychological test data to optimize the patient's strengths in treatment. An objective brain-to-behavior approach allowed the patient to engage in treatment modalities specific to her strengths, leading to demonstrable improvement over the course of her most recent interventions.

\section{REFERENCES}

Amen, D. G. \& Carmichael, B. D. (1997). High resolution brain SPECT imaging in ADHD. Annals of Clinical Psychology, 9(2), 81-86.

American Psychiatric Association. (2000). Diagnostic and statistical manual of mental disorders $\left(4^{\text {th }}\right.$ ed., Text Revision). Washington, DC: Author.

Baerwald, J.P., Tryon, W.W. \& Sandford, J. (2001). Modal Attention Asymmetry in Patients with Schizophrenia, Bipolar Disorder, as Compared to Normal Controls. neuropsychology, 15(4), 535 543.

Bentaleb, L., Beauregard, M., Liddle, P., \& Stip, E. (2002). Cerebral activity associated with

auditory verbal hallucinations: A functional magnetic resonance imaging case study. Journal of Psychiatry \& Neuroscience, 27(2), 110-115.

Congedo, M., \& Lubar, J. F. (2003). Parametric and non-parametric analysis of QEEG: Normative database comparisons in electroencephalography, a simulation study on accuracy. Journal of Neurotherapy, 7(3-4), 1-29. doi:10.1300/J184v07n03_01

Dickerson, F., Boronow, J. J., Stallings, C., Origoni, A. E., Cole, S. K., \& Yolken, R. H. (2004). Cognitive functioning in schizophrenia and bipolar disorder: Comparison of performance on the Repeatable Battery for the Assessment of Neuropsychological Status. Psychiatry Research, 129(1), 45-53. doi:10.1016/ j.psychres.2004.07.002

Ford, J. M., Roach, B. J., Jorgensen, K. W., Turner, J. A., Brown, G. G., Notestine, R. et al. (2009). Tuning in to the voices: A multisite fMRI study of auditory hallucinations. Schizophrenia Bulletin, 35(1), 5866.

Goethals, I., Audenaert, K., Jacobs, F., Van den Eynde, F., Bernagie, K., Kolindou, A., et al. (2005). Brain perfusion SPECT impulsivity-related personality disorders. Behavioral Brain Research, 157(1), 187-192.

Goforth, H. W., Konopka, L., Primeau, M., Ruth, A., O’Donnel, K., Patel, R. et al. (2004). Quantitative electroencephalography in frontotemporal dementia with methylphenidate response: A case study. Clinical EEG and Neuroscience, 35(2), 108-111.

Goldenberg, G., Podreka, I., Steiner, M., Franzen, P., \& Deecke, L. (1991). Contributions of occipital and temporal brain regions to visual and acoustic imagery-A SPECT study. Neuropsychologia, 29(7), 695702.

Hughes, J. R., \& John, E. (1999). Conventional and quantitative electroencephalography in psychiatry. The Journal of Neuropsychiatry and Clinical Neurosciences, 11(2), 190-208.

Ishii, R., Shinosaki, K., Ikejiri, Y., Ukai, S., Yamashita, K., Iwase, M. et al. (2000). Theta rhythm increases left superior temporal cortex during auditory hallucinations in schizophrenia: A case report. NeuroReport, 11(14), 3283-3287.

Kane, H. \& Whiston, S.C. (2001). Review of the IVA Continuous Performance Test. Buros Fourteenth Mental Measurements Yearbook, 592-595.

Kim, B., Lee, J., Shin, M., Cho, S., \& Lee, D. (2002). Regional cerebral perfusion abnormalities in attention deficit/ hyperactivity disorder: Statistical parametric mapping analysis. European Archives of Psychiatry and Clinical Neuroscience, 252(5), 219-225. doi:10.1007/ s00406-002-0384-3

Lawrence, A.D. \& Sahakian, B.J., Eds. 1996. The neuropsychology of frontostriatal dementias. In: Woods, R.T., Editor, 1996. Handbook of the Clinical Psychology of Aging, Wiley, New York, pp. 243-265.

Lennox, B. R., Park, S. B. G., Medley, I., Morris, P. G., \& Jones, P. B. (2000). The functional anatomy of auditory hallucinations in schizophrenia. Psychiatry Research: Neuroimaging 100, 13-20.

Lezak, M. (1995). Neuropsychological assessment (3rd ed.). New York, NY US: Oxford University Press. 
Mauri, M. C., Gaietta, M., Dragogna, F., Valli, I., Cerveri, G., \& Marotta, G. (2008). Hallucinatory disorder, an original clinical picture? Clinical and imaging data. Progress in NeuroPsychopharmacology \& Biological Psychiatry, 32, 523-530.

McGuire, P. K., Silbersweig, D. A., \& Frith, C. D. (1996). Functional neuroanatomy of verbal selfmonitoring. Brain, 119, 907-917.

Poprawski, T.J., Pluzyczka, A.N., Park, Y., Chennamchetty, V.N., Halaris, A., Crayton, J.W., Kon poka, L.M. (2007). Multimodality imaging in a depressed patient with violent behavior and temporal lobe seizures. Clinical EEG and Neuroscience, 38 (3), 175-179.

Reitan, R. M. \& Wolfson, D. (1986). The Halstead-Reitan neuropsychological test battery and aging. Clinical Gerontologist: The Journal of A ging and Mental Health, 5(1-2), 36-61. doi:10.1300/ J018v05n01_03

Robbins, T.W., James, M., Owen, A.M., Sahakian, B.J., McInnes, L. \& Rabbitt, P. (1994). Cambridge Neuropsychological Test Automated Battery (CANTAB): A factor analytic study of a large sample of normal eld erly volunteers. Dementia, 5, 266-281

Spencer, K. M., Niznikiewicz, M. A., Nestor, P. G., Shenton, M. E., \& McCarley, R. W. (2009). Left auditory cortex gamma synchronization and auditory hallucination symptoms in schizophrenia. BMC Neuroscience, 10(85), 1-13.

Sweeney, J. A., Kmiec, J. A., and Kupfer, D. J. (2000). Neuropsychologic impairments in bipolar and unipolar mood disorders on the CANTAB neurocognitive battery. Biological psychiatry, 48 (7), 674684. doi:10.1016/ S0006-3223(00)00910-0

Thatcher, R. W., Walker, R. A., Biver, C. J., North, D. N., \& Curtin, R. (2003). Quantitative EEG normative database: Validation and clinical correlation. Journal of Neurotherapy, 7 (3-4), 87-121. doi: 10.1300/ J184v07n03_05

Tiijhonen, J., Hari, R., Naukkarinen, H., Rimon, R., Jousmaki, V., \& Kajola, M. (1992). Modified activity of the human auditory cortex during auditory hallucinations. American Journal of Psychiatry, 149(2), 255-257.

van de Ven, V. G., Formisano, E., Roder, C. H., Prvulovic, D., Bittner, R. A., Dietz, M. G., et al. (2005). The spatiotemporal pattern of auditory cortical responses during verbal hallucinations. NeuroImage, 27, 644-655. 